\title{
A Novel Lips Detection Method Combined Adaboost Algorithm and Camshift Algorithm
}

\author{
Wen Feng \\ Chongqing University of Posts and Telecommunications \\ Chongqing China \\ zhouyw@cqupt.edu.cn
}

\begin{abstract}
The lips detection has a very unique role in identification and tracking, voice recognition, lipsreading and so on. This paper proposed a new lips detection method by combined Adaboost algorithm and Camshift algorithm. First, detects the lips by the lips classification which trained by Adaboost algorithm, and marks the specific lips location with a rectangular window. Then, makes the lips color region as the tracking color tracked by Camshift algorithm, and track the lips position in the next frame. Finally, uses Adaboost algorithm to do lips detection. The new lips detect method overcomes search the entire window just use Adaboost algorithm, greatly improved the accuracy of lips detection.
\end{abstract}

Keywords-component, fAdaboost algorithm, Camshift algorithm, lips detection

\section{INTRODUCTION}

Face features extraction are important issue in face recognition, face recognition, human-computer interaction and other areas. Face features, such as eyebrows, pupils, eyelids, lips are great significance in face recognition. Especially, the lips detection has a very unique role in identification and tracking, voice recognition, lips-reading and so on. The lips detection has many methods, but there are some deficiencies: HungYong-hui, Pan Baa-chang, applied Haar-features to train Adaboost classifiers, and combined skin and lips color separation algorithm forms a self-adaptive skin and lips color separation model to detect the lips, but the correct rate would drop in the detection of different colors lips[1].Chih Yu Hsu, Chih-Hung Yang proposed a method for lips recognition based on Active Basis Model (ABM).This method involved the SVM to learn the lips classifiers, but this algorithm have the expensive computation and poor real time[2].Yong hui huang, Bao chang pan proposed a detection and localization in lips-reading system based on least square fitting and two stage ellipses fitting, but the threshold is difficult to determine on edge detection, and lead to great differences on test results [3].Xu cheng-yang and PRINCE J L use the classical Snake model to detect the mouth area, but there are some weakness on mouth edge, and difficult to separate them accurately by classical Snake model[4].

This paper proposed a new lips detection method by combined Adaboost algorithm and Camshift algorithm. First, detects the lips by the lips classification which trained by Adaboost algorithm, and marks the specific lips location with a rectangular window. Then, makes the lips color region as the tracking color tracked by Camshift algorithm, and track the lips position in the next frame. Finally, uses Adaboost algorithm to do lips detection. The new lips detect method overcomes search the entire window just use Adaboost algorithm, greatly improved the accuracy of lips detection.

\section{ADABBOST ALGORITHM AND LIPS DETECTION}

\section{A. Haar-like rectangular character}

Haar-like features used by the Papageorgiou for face detection firstly, then Viola proposed three kinds and four forms of the characters. Three types are: 2 - rectangular character, 3 - rectangular character, 4- rectangle character, four forms are shown in Figure 1 [5].

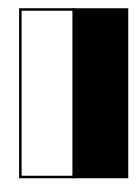

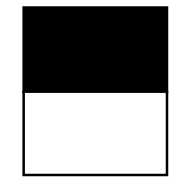

A

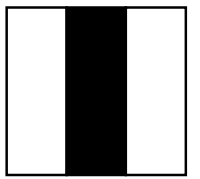

C D

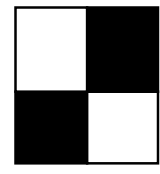

Figure 1. Haar-like character
The haar-like character is defined as the sum of the pixels which lie within the white rectangles subtracted from the sum of pixels in the gray rectangles. Different characters can be interpreted as calculate a directional reciprocal to a set of rectangle character points correspond, to obtain the additional point sets information to provide a base for the lips detection.

Adabbost algorithm can identify specific areas in the limited data situation, it uses the rectangular character ( haar-like character )as lips character vector, that is 
because the haar rectangular characters are sensitive for some simple graphics such as edges, lines. So the lips character can be simply described by the rectangular character.

\section{B. Lips detection classifier}

In this paper, the Haar-like features proposed by Voila are used to form the weak lips classifiers. First, $\mathrm{N}$ training samples which have K character values are collected, each character value has a weak classifier, the weak lips classifier of the $\mathrm{j}$ eigenvalue form is expressed as equation(1) [6]:

$$
h_{j}(x)= \begin{cases}1 & P_{j} f_{j}(x)<P_{j} \theta_{j} \\ 0 & \text { otherwise }\end{cases}
$$

$h_{j}(x)$ is the value of the weak classifier, 1 are lips, 0 are non-lips, $\theta_{j}$ is the threshold of the weak classifier, $P_{j}$ is the direction of inequality, its Value is -1 or +1 , if the lips samples are classified below the threshold, $P_{j}=+1$, otherwise,$P_{j}=-1 ; x$ is the sub-window to be detected, $f_{j}(x)$ is the eigenvalue.

After obtaining the weak lips classifiers, choose $\mathrm{T}$ optimal weak lips classifiers, and optimize them into a strong lips classifier, the final strong lips classifier is expressed as equation(2) [7]:

$$
h(x)= \begin{cases}1 & \sum_{t-1}^{T} a_{t} h_{t}(x) \geq 1 / 2 \sum_{T-1}^{T} a_{t} \\ 0 & \text { otherwise }\end{cases}
$$

$a_{t}=\log \frac{1}{\beta_{t}}, \beta_{t}=\frac{\varepsilon_{t}}{1-\varepsilon_{t}}, \varepsilon_{t}$ is the smallest error in all the Weak lips classifiers.

\section{Adabbost algorithm detect lips}

Adabbost algorithm is one kind of self-adaptation boosting algorithms. Using this algorithm, the multi weak learner is boosted into a strong one, lips detection using Adabbost algorithm basic philosophy is described as following: given a weak lips classification algorithm and a lips training sets $\{(\mathrm{x} 1, \mathrm{y} 1),(\mathrm{x} 2, \mathrm{y} 2), \ldots,(\mathrm{xn}, \mathrm{yn})\}, \mathrm{xi}$ is the input of lips training samples, yi is the classify categories, yi $\{0,1\}, 1$ is lips, 0 is non-lips, respectively. All the lips training samples are assigned to same weight $\mathrm{D}=1 / \mathrm{n}$ initially, then Adabbost calls the lips weak classification algorithm in a series of rounds $\mathrm{T}=1,2, \ldots$, repeatedly. For each calling, a distribution of weight $D_{t}$ is updated, that indicates the importance of examples in the data sets for the classifiers. On each round, the weights of each incorrectly classified lips example are increased (or alternatively, the weights of each correct classified lips example are decreased), so that the new lips classifier focuses more on those examples. When the lips classifier distributes certain lips sample correctly, the weight of these lips samples will be reduced. When misclassified, the weights of these lips samples will be increased to make the learning algorithm concentrate to carry on the quite difficult lips training sample to learn in the following study. According to the weighted voting's way, we merge the weak lips rule which produces each wheel to be a strong one finally[7], and then a lips detection cascade classifier is built by Adabbost algorithm .Obtain the lips rectangular template by lips classifier first, and then use the template to match the detected lips. Figure 2 is the lips detect diagram block [8].

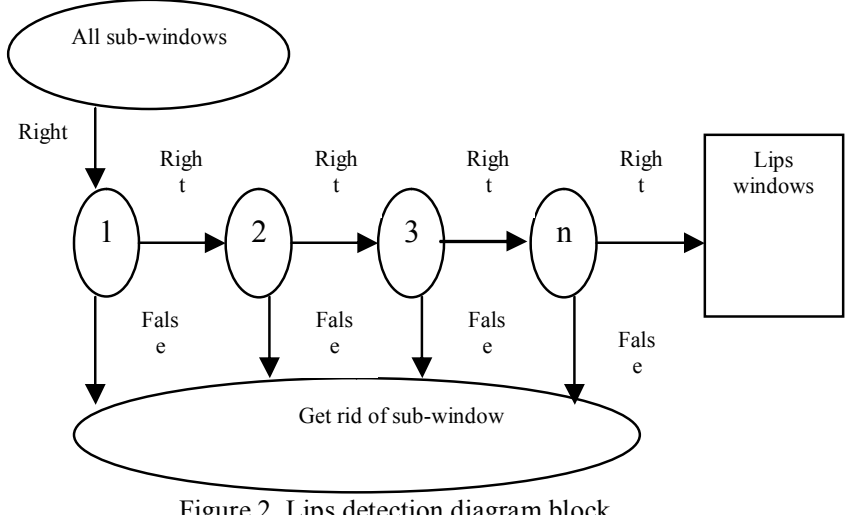

III. THE LIPS TRACKING THEORY OF CAMSHIFT ALGORITHM

The Camshift lips tracking algorithm can be divided into four basic steps[9.10]:

1) Initialize a search window $W$ in color probability distribution, the size is $\mathrm{S}$;

2)"Convergence" search window by using the Mean Shift algorithm;

$Z_{00}$ is given by the following formula:

$$
\begin{aligned}
& Z_{00}=\sum_{x} \sum_{y} p(x, y) \\
& Z_{10}=\sum_{x} \sum_{y} p(x, y)(a) \\
& Z_{01}=\sum_{x} \sum_{y} p(x, y)(1.2 a)
\end{aligned}
$$

the centroid of search window $\left(x_{0}, y_{0}\right)$ :

$$
x_{0}=\frac{Z_{10}}{Z_{00}}(a), y_{0}=\frac{Z_{01}}{Z_{00}}(1.2 \mathrm{a})
$$

Use the center of the search window to compute the centroid. Repeat the process until "convergence."

3) Reset the size $\mathrm{S}$ of search window, and calculate the output parameters of tracking target;

Update the width of search window: 


$$
a=2 * \sqrt{\frac{Z_{00}}{256}}
$$

4) Jump to the second step to do the cycle of next frame.

\section{EXPERIMENTAL RESULTS}

\section{A System hardware}

The main hardware selections and their functions described as following:

1) Camera: Teclast high-definition network video camera Transformers T838.

2) Image processor: Implement lips detection, analyzing and processing the acquired image sequences to achieve the lips detection. The system uses a laptop computer with the Windows XP operating system as image processor.

\section{$B$ System shoftware}

The system uses $\mathrm{VC}++6.0$ and $\mathrm{OpenCV}$ as a software program environment. OpenCV is consisted of $\mathrm{C}$ functions and $\mathrm{C}++$ class library, including image processing, computer vision, and pattern recognition. It provides a large number of general algorithms, involving image processing (the difference of image, the convert from RGB image to gray image, binary processing, they can directly call the functions to achieve these functions), structure analysis, motion detection, camera calibration, 3-dimensional reconstruction, machine learning and so on, and has a high learning efficiency[11].Figure 3 is the lips detection block diagram by Adabbost and Camshift algorithm

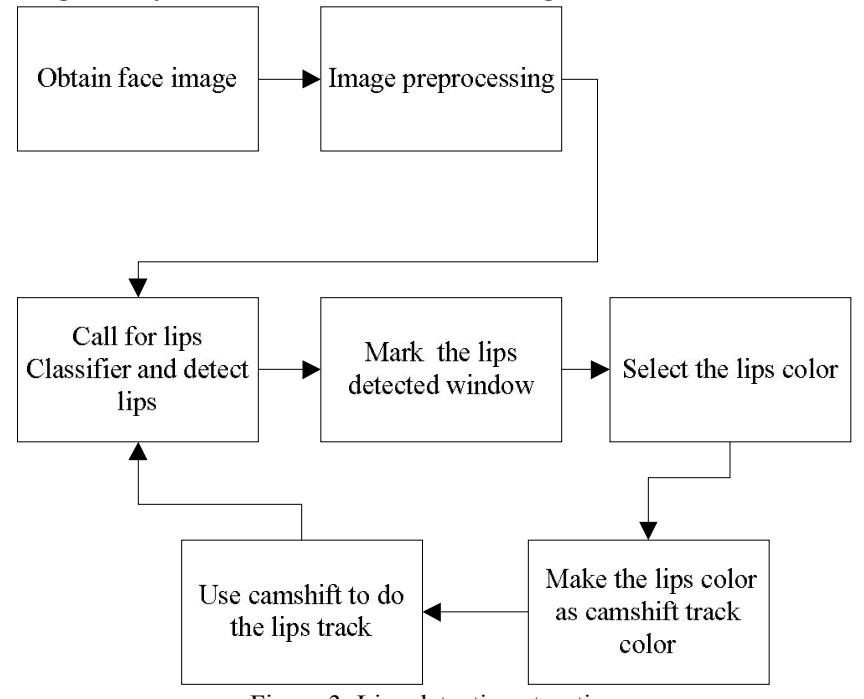

Figure 3. Lips detection struction

The detailed explanation of the figure 3 as following, get the face image and preprocess the face image, the preprocessing include: color conversion, histogram equalization, illumination compensation. Call the pre- trained lips classifiers to detect lips and mark the lips location by a rectangular window, make the lips color region as the Camshift algorithm tracking color, and tracks the position of lips window in the next frame. Feed back the position to the lips classifier to detect lips in the next frame.

\section{Experimental results analysize}

Acquire video image through the USB camera, catch each frame from the video images. In order to reduce the influence of light during the image detection, all the snatched images are histogram equalized (this is image preprocessing). Call the lips cascade classifiers trained by Adabbost algorithm to detect the lips, mark the specific location of the lips with a rectangular window. Then, make the lips color region as the Camshift algorithm tracking color, and tracks the position of lips window in the next frame. Finally, feed back the tracking results to the lips classifier to detect the lips of image in the next frame.

The specific detection process are as follow: Set the initial search window for lips $25 \times 15$ which is moved in the detected window, the search window detects every possible position to determine the lips position, the lips are marked with a green rectangular window after detected. Then, make the lips color region as the Camshift algorithm tracking color, and tracks the position of lips window in the next frame, and feedback the tracking results to the lips classifier to detect the lips in the next frame.The real-time and correct rate of the lips detection have very good improvement by using this lips detection method.

The following is the test results of images with human face that are captured by the camera in one second. The paper grab one frame every five frames. The specific test results as figure 4 :
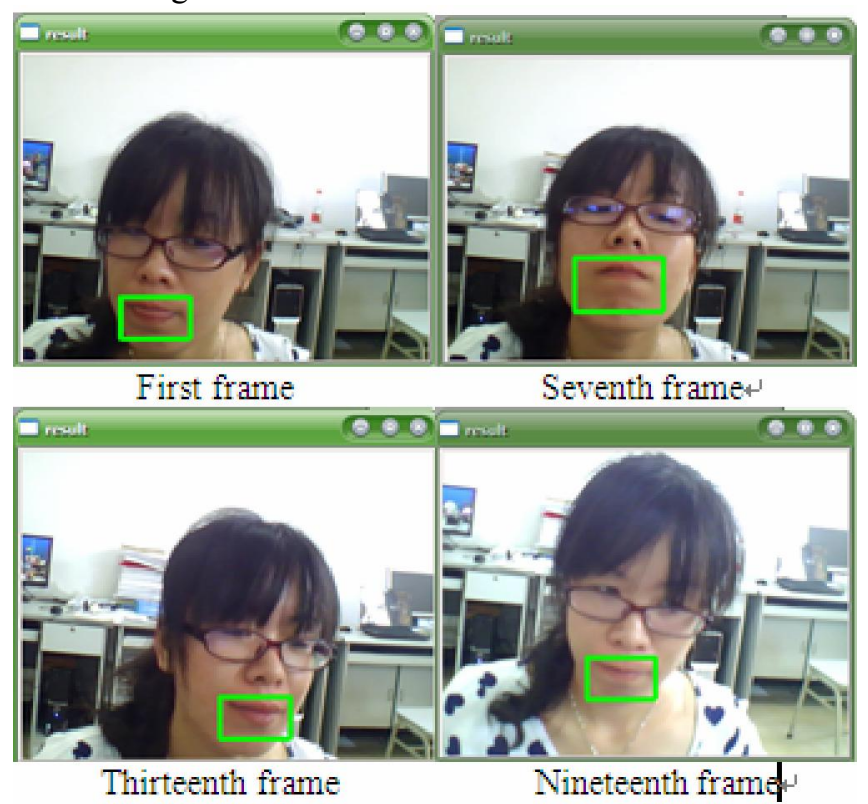

Figure 4. Experiment of lips detection results 
From the above experimental results show that the different lips posture are detected well by using the combined lips detection method of Adabbost and Camshift algorithms.

In order to verify the correct rate of this new lips detected algorithm on lips detection further, this paper did 100 groups of experiments, each group of experiments detected 1500 images. Here are just the three groups of experimental results:

TABLE I THE EXPERIMENTAL RESULTS OF THE NEW LIPS DETECT ALGORITHM

\begin{tabular}{|l|l|l|l|}
\hline & Correct rate & Error rate & Undetected \\
\hline 1 & $91.3 \%$ & $6.9 \%$ & $1.8 \%$ \\
\hline 2 & $89.7 \%$ & $7.1 \%$ & $3.2 \%$ \\
\hline 3 & $92.8 \%$ & $5.3 \%$ & $1.9 \%$ \\
\hline Average & $91.27 \%$ & $6.43 \%$ & $2.3 \%$ \\
\hline
\end{tabular}

From the experimental results show that, the lips detection correct rate has achieved $91.27 \%$ by using the lips detection method of the combined Adaboost and Camshift algorithms, and the lips detection average error rate was just $6.43 \%$ by using the new combined algorithm, and the experiment fully proved the effectiveness of the new algorithm.

\section{CONCLUSION}

This paper proposed a new lips detection method combined the Adaboost algorithm Camshift algorithm, and did a lot of experiments. Experimental results show that by combining the Adaboost algorithm Camshift algorithm to do detect the lips, the detection results has a good effect, the robustness of the lips detection is improved.

\section{REFERENCES}

[1] HUANG Yong-hui, PAN Baa-chang LIANG Jian .A new lipsautomatic detection and location algorithmin lips-reading system[C], Systems Man and Cybernetics (SMC), 2010 IEEE International Conference on, Istanbul ,10-13,Oct,2042-2045.

[2] Chih-Yu Hsu, Chih-Hung Yang, Yung-Chih Chen, Min-chian Tsai. A PSO-SVM Lips Recognition Method Based on Active Basis Model[C], 2010 Fourth International Conference on Genetic and Evolutionary Computing,Shenzhen,China,July,2010,743-747.

[3] Yong-hui huang, Bao-Chang Pan , Sheng-Lin Zheng ,Jianjia Pan, Yuanyan Tang. Lips-reading detection and localization based on two stage ellipse fitting[C], Proceedings of the 2008 International Conference on Wavelet Analysis and Pattern Recognition, Hong Kong, 30-31 Aug. 2008.168-171.

[4] XU Cheng-yang, PRINCE J L. Snakes, shapes and Lang Li-ying, Gu Wei-wei." Improved Face Detection Algorithm Based on Adaboost" [C].2009 International Conference on Electronic Computer Technology, 2009, Page(s): $183-186$

[5] JosC Barreto, Paulo Menezest and Jorge Diad. "Human-Robot Interaction based on Haar-like Features and Eigenfaces"[C]. ICRA'04.2004 IEEE International Conference on Robotics and Automation, 2004, shanghai,China,May,Page(s): 1888 - 1893 Vol.2.

[6] Baoguo Wei, Jing Li.Object Tracking via Modified Camshift in Sequential Bayesian Filtering Framework[C], 2010 3rd International Congress on Image and Signal Processing (CISP2010),oct1820, Shandong,yantai,358-362.

[7] Xian WU Lihong LI Jianhuang LAI ? Jian HUANG. A Framework of Face Tracking with Classification using Camshift-C and LBP[C], 2009 Fifth International Conference on Image and Graphics, Sep. 2124, shanxi, Xi'an 217-222.

[8] Ruizhen Liu, Shiqi Yu. "OpenCV Tutorial" [M].Beijing: Beijing University of Aeronautics and Astronautics publishing house, 2008. 